\title{
Miniaturized multi-channels SiPM read-out electronics for medical imaging application
}

\author{
T. Ait Imando', N. Dinu', S. Callier ${ }^{1}$, D. Cuisy ${ }^{1}$, M. Gaspard', \\ L. Pinot ${ }^{2}$, V. Puill ${ }^{1}$, L. Raux ${ }^{1}$, S. Trochet ${ }^{1}$, L. Menard ${ }^{2}$ \\ ${ }^{1}$ Laboratory of Linear Accelerator (LAL), IN2P3-CNRS, 91898 Orsay, France \\ ${ }^{2}$ Laboratory of Imaging and Modeling in Neurobiology and Oncology (IMNC), IN2P3-CNRS, 91406 \\ Orsay, France
}

Corresponding author: imando@lal.in2p3.fr

The SIPMED is a prototype of a very compact intra-operative gamma camera based on arrays of Silicon Photomultiplier (SiPM) coupled to a scintillator and dedicated read-out electronics to improve the efficiency and the reliability of the image guided surgery of tumors. The SIPMED consists of $2 \times 2$ elementary modules covering a field of view of $5 \times 5 \mathrm{~cm}^{2}$ and having geometrical dimensions adapted for intra-operative interventions ( $\sim 6 \mathrm{~cm}$ width, $\sim 5 \mathrm{~cm}$ thickness and weight less than $700 \mathrm{gr})$. Each elementary module is composed of a very compact stack of three different PCBs.

$2 \times 2$ monolithic SiPM arrays of 16 channels each are mounted on the first PCB [1]. The two readout chips EASIROC of 32 channels are directly bonded on the second PCB [2]. The FPGA and the USB interface are mounted on the third PCB. The SIPMED camera will be composed of 256 read-out channels.

International Workshop on New Photon-detectors,

LAL Orsay, France

June 13-15, 2012

1 Poster 


\section{Introduction}

The precise localization and complete surgical excision of tumors is one of the most important procedures in the cancer treatment. The aim of the surgery is to process a total resection of the tumors mass while sparing surrounding normal tissues. However, the precise delineation of the tumor extend beyond the visible boundary of the nodes is a challenging procedure. In that context, intra-operative imaging of tumors labeled with radiotracers is more and more used to guide the surgeon during excision.

The key parameters when trying to improve the efficiency of intra-operative beta or gamma probes for real-time tumor localization are sensitivity, compactness, biocompatibility and cost. The new generation of semiconductor photon-detector called Silicon Photomultiplier (SiPM) present important advantages like good photon-detection efficiency, compactness, lightness and low bias voltage. Consequently, it has the potential to fulfill all the mentioned needs for building the intra-operative probes and to overcome the main drawbacks of current technologies.

However, further developments are still necessary to achieve a reliable compact photondetection system that could impact the performances of future intra-operative devices. In particular, arrays of SiPM's have to be used to obtain a large field-of-view as well as a miniaturized electronics based on a dedicated ASIC has to be developed for the multi-channels SiPM arrays read-out. The design of such a photo-detection system developed at LAL is presented in the following.

Our goal, in term of miniaturization of the electronic readout ( 64 channels), is to match the size of an array of $2 \times 2$ 16-channel monolithic SiPM matrix with $3 \times 3 \mathrm{~mm}^{2}$ elements. The compact package of electronics will enable to assemble side-by-side several elementary arrays to obtain photosensors with various fields of view.

\section{Elementary module of the SIPMED gamma camera}

The photo-detection SiPM system is composed by a compact stack of three PCBs which deliver the high voltage, dispatch and readout the output signals. The first board contains the four monolithic SiPM arrays on one side and a digital thermal sensor on the other side. The second board contains two ASIC's named EASIROC, each of them having 32 channels and allowing individual connection of the SiPM bias voltage using the chip embedded DAC in order to maintain a constant gain on each SiPM during the system operation, two external 12-bit ADCs allowing ASIC data acquisions, for Low Gain and High Gain. The third board contains an FPGA for treatment of the SiPM's output signals and a DC/DC Converter to deliver the high voltage.

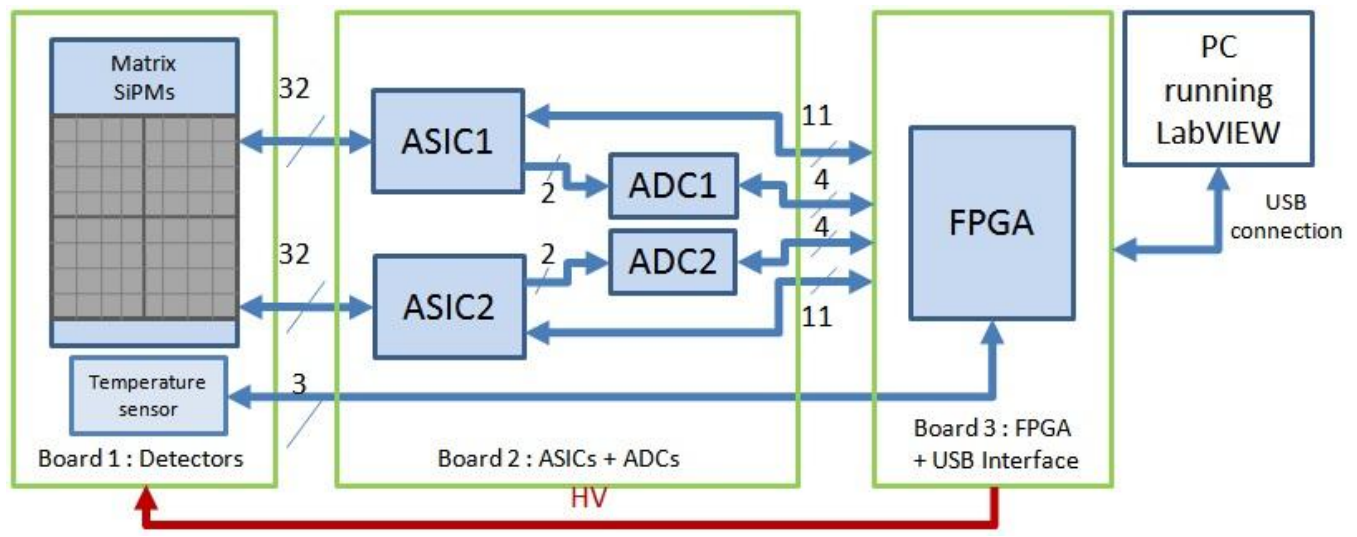

Figure 1 : Block diagram 
A picture of the photo-detection SiPM system composed of three boards stacked together $\left(28.6 \times 27.2 \mathrm{~mm}^{2}\right)$ is presented in Figure 2. Detailed pictures of separate boards are presented in Figure 3.

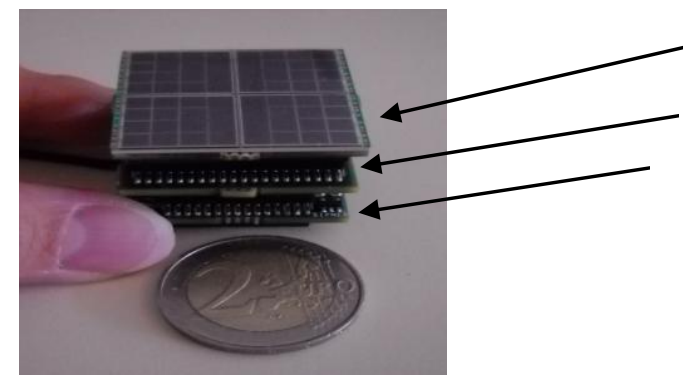

SiPM board

ASIC board

FPGA board

Figure 2: compact stack

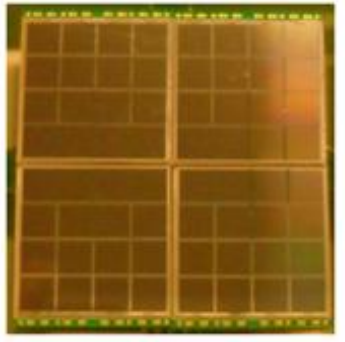

a)

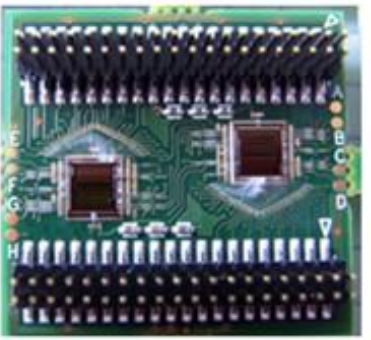

b)

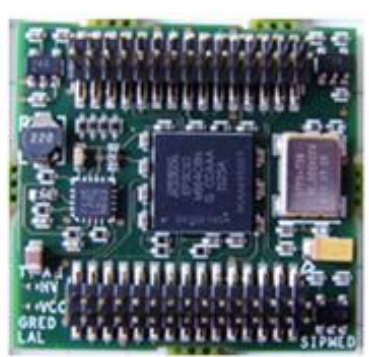

c)

Figure 3: SiPM board (a), ASIC board (b) and FPGA board (c)

\section{Measurements}

Each elementary module is tested individually, without SiPM board, with dedicated software (LabVIEW using USB interface) to test and to characterize the ASICs. The main points of interests are: DACs linearity, channels uniformity and $100 \%$ trigger efficiency.

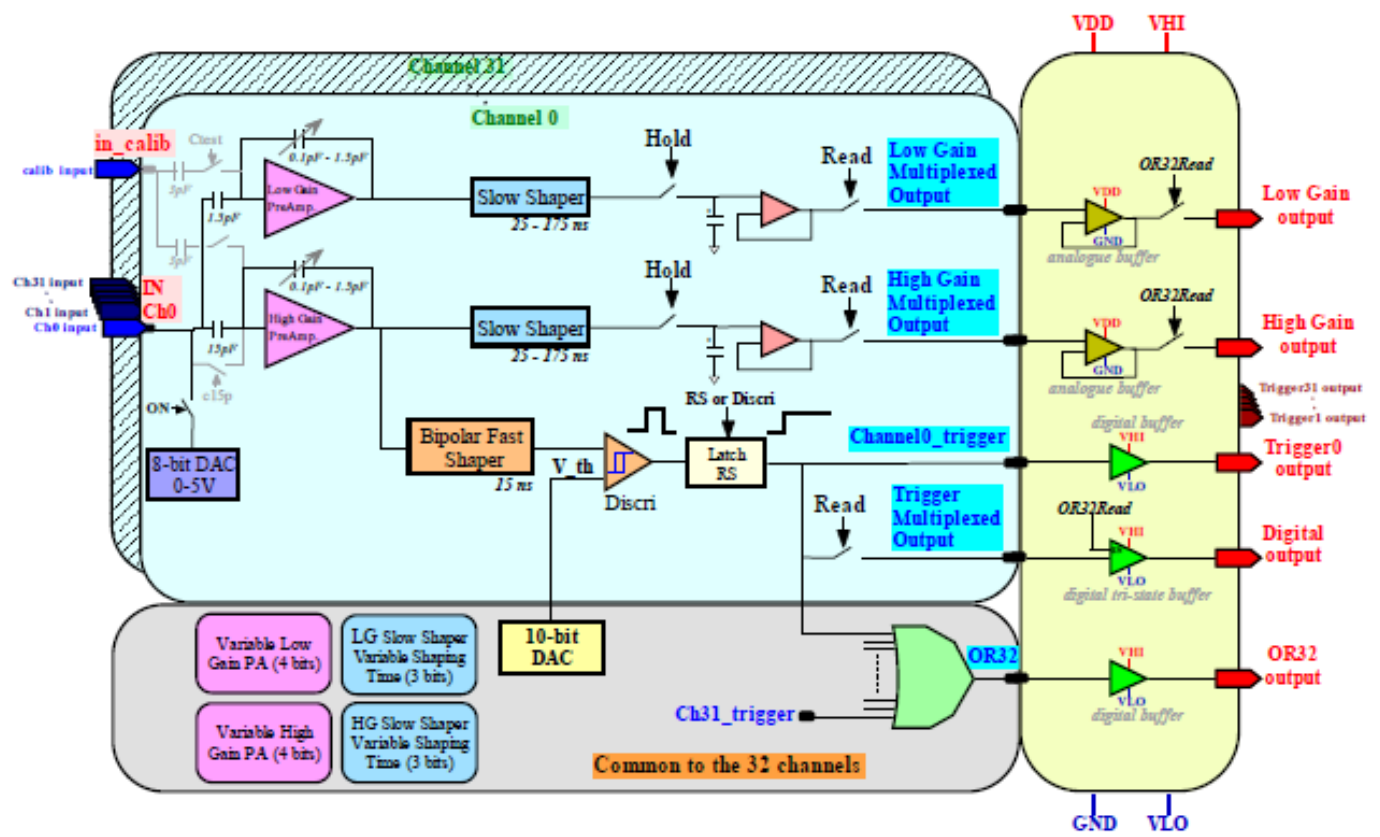

Figure 4: ASIC schematic 


\subsection{8-bit input DAC performance}

The input DAC span goes from $2.5 \mathrm{~V}$ down to $0 \mathrm{~V}$. The default value is $2.5 \mathrm{~V}$ in order to operate the SiPM at minimum over-voltage when the DAC is not loaded. The linearity is $\pm 2 \%$, just enough for the SiPM operation.
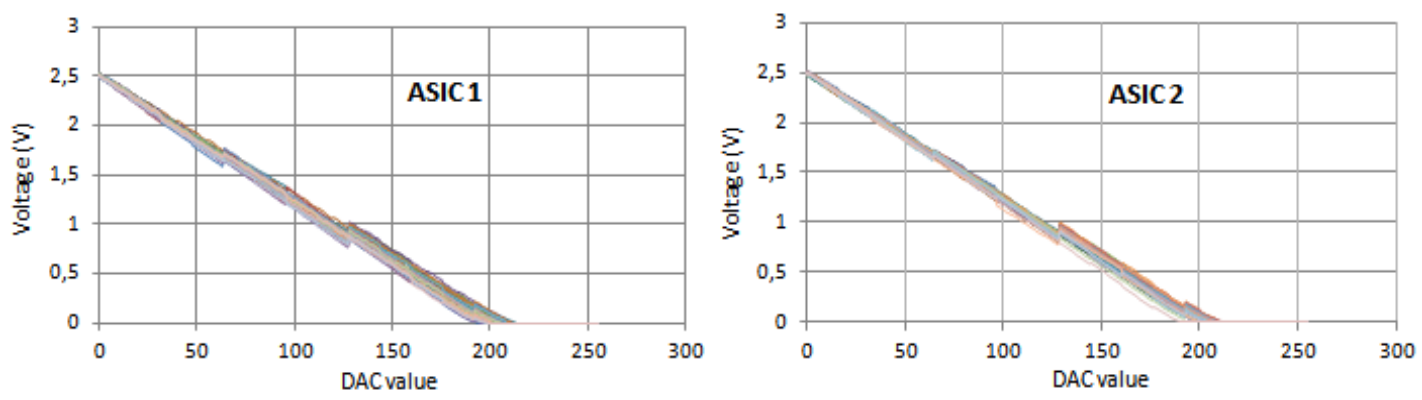

Figure 5: 8-bit DAC linearity

\subsection{0-bit DAC measurement}

A dedicated $15 \mathrm{~ns}$ fast shaper is following the high gain preamplifier. This outputted signal is then compared to a threshold, thanks to a discriminator which provides the trigger signal, which can be latched. Any channel trigger can be quiet. The threshold is common for all the 32 channels for each ASIC and is set by a 10-bit DAC. This DAC has $1.3 \mathrm{mV}$ steps and allows threshold tuning from $1 \mathrm{~V}$ up to $2.4 \mathrm{~V}$.

The DAC linearity was checked by scanning all the values and measuring the signal for each combination. The figure below gives the evolution of the signal amplitude as a function of the DAC combination. By fitting this line in the region without saturation, we obtained a nice linearity of $\pm 0.2 \%$ on a large range.

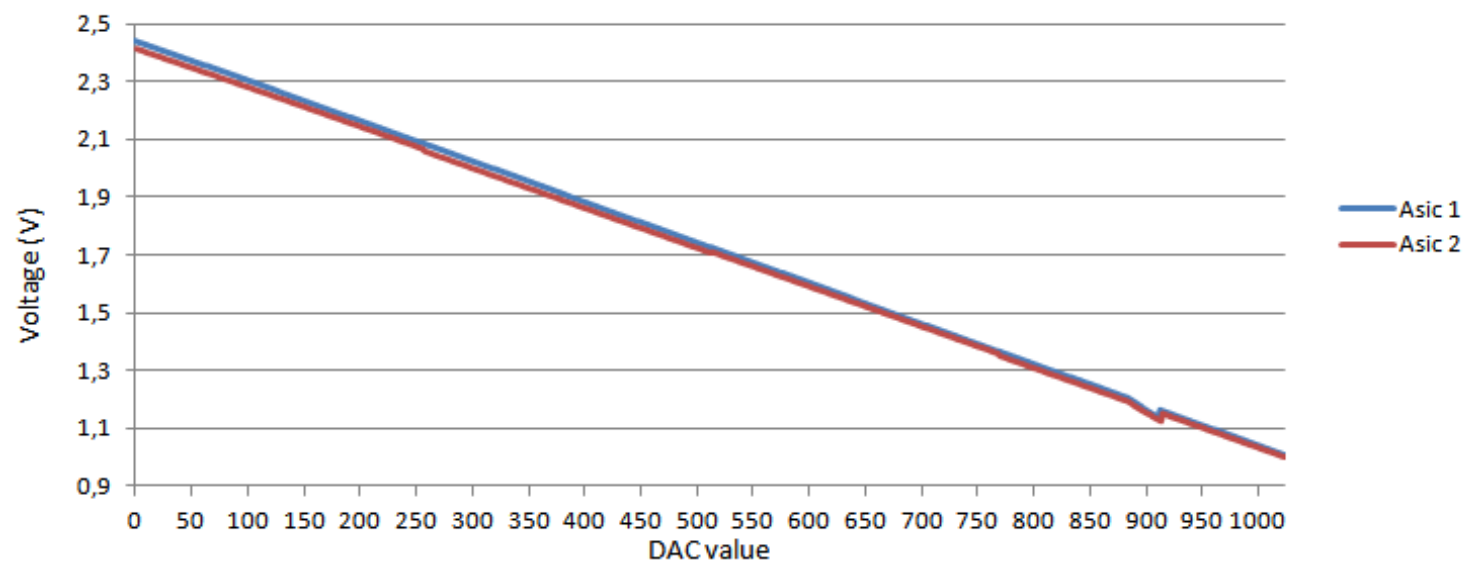

Figure 6: 10-bit DAC linearity

\subsection{Charge measurement}

EASIROC has two analogue outputs, one for Low Gain, one for High Gain. Each is digitized using an ADC. Like an example, we present below a waveform of slow shaper high gain (HG) output for a fixed injected charge of $1.6 \mathrm{pC}$ on channel 10, and the live ADC data acquisition for each channel. 

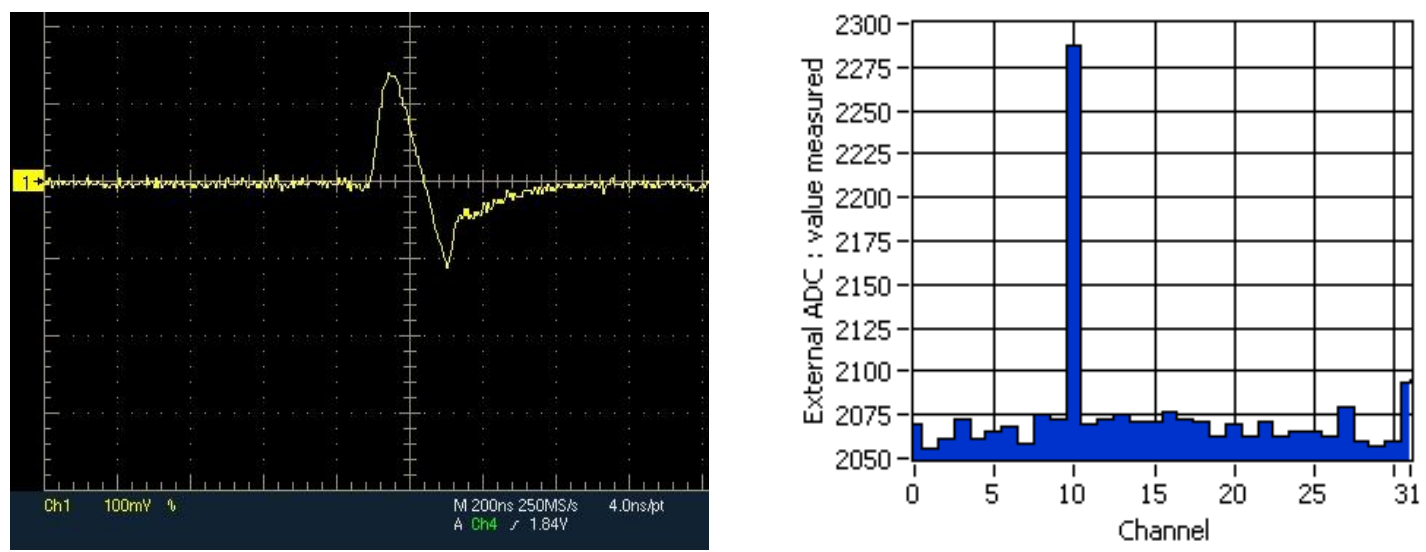

Figure 7: Scope screenshot of slow shaper HG output for a fixed injected charge of $1.6 \mathrm{pC}$ (left) and the corresponding live ADC acquisition (right)

Waveforms were recorded with a fixed injected charge of 640fC and for variable preamplifier gains as one can see on the Figure 8 which represents the amplitude as a function of feedback capacitance (Cf) for different gains. The fit of the linear part of the curve is better than $1 \%$. The next figure represents the high gain digital output signal amplitude as a function of the injected charge.

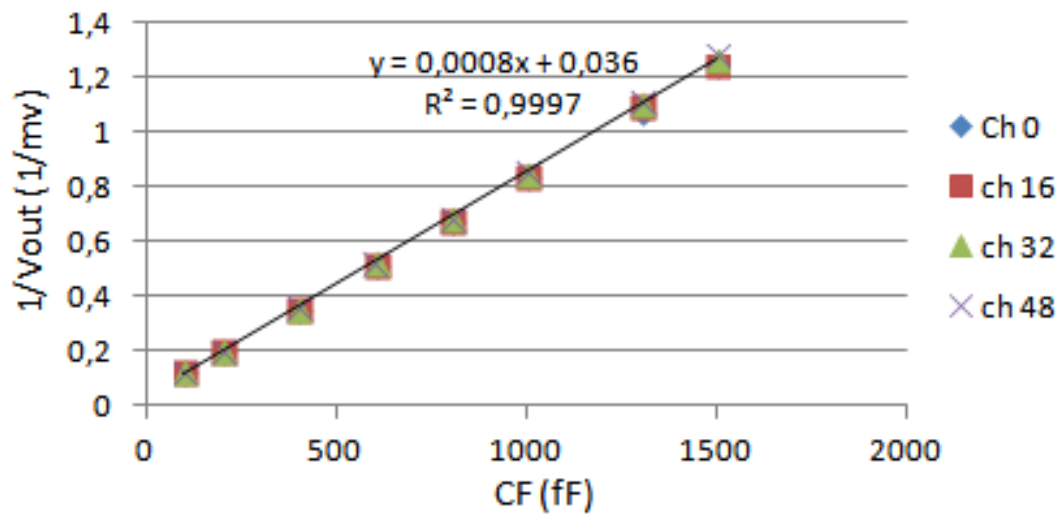

Figure 8: 1/Vout versus Cf

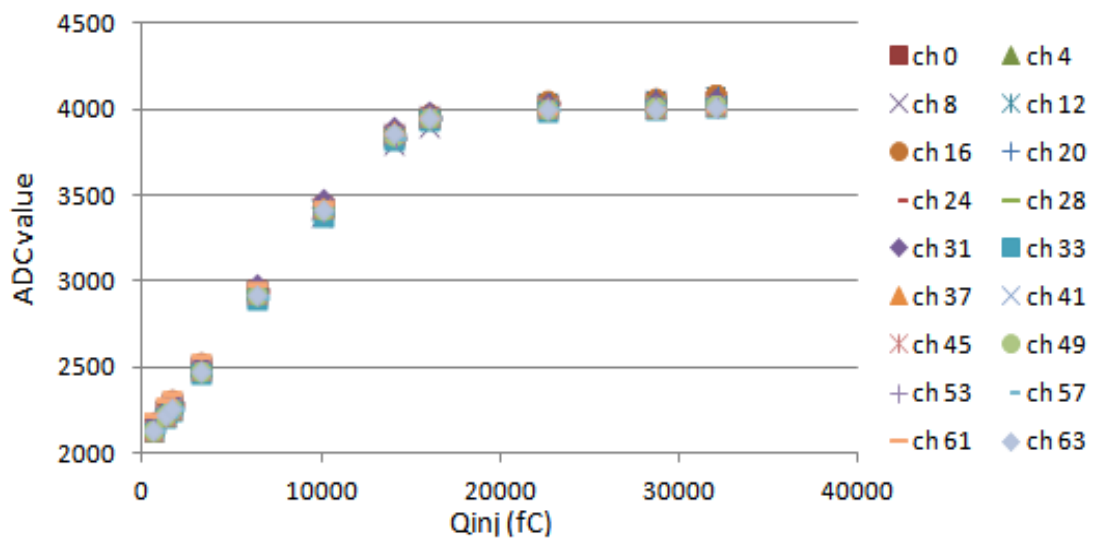

Figure 9: high gain versus injected charge 


\subsection{Trigger efficiency measurement}

S-curves correspond to the measurement of the trigger efficiency during a scan of the input charge or the threshold while the other parameters, like the preamplifier gain, are kept constant. Figure 10 represents the trigger efficiency as a function of the DAC values for the 32 channels of each ASIC. Channel 31 was set at $\mathrm{Cf}=200 \mathrm{fF}$ and the input signal was fixed at Qinj=160fC. We obtained $100 \%$ trigger efficiency for an input charge of approximately $160 \mathrm{fC}$ which corresponds to 1 pe as requested.

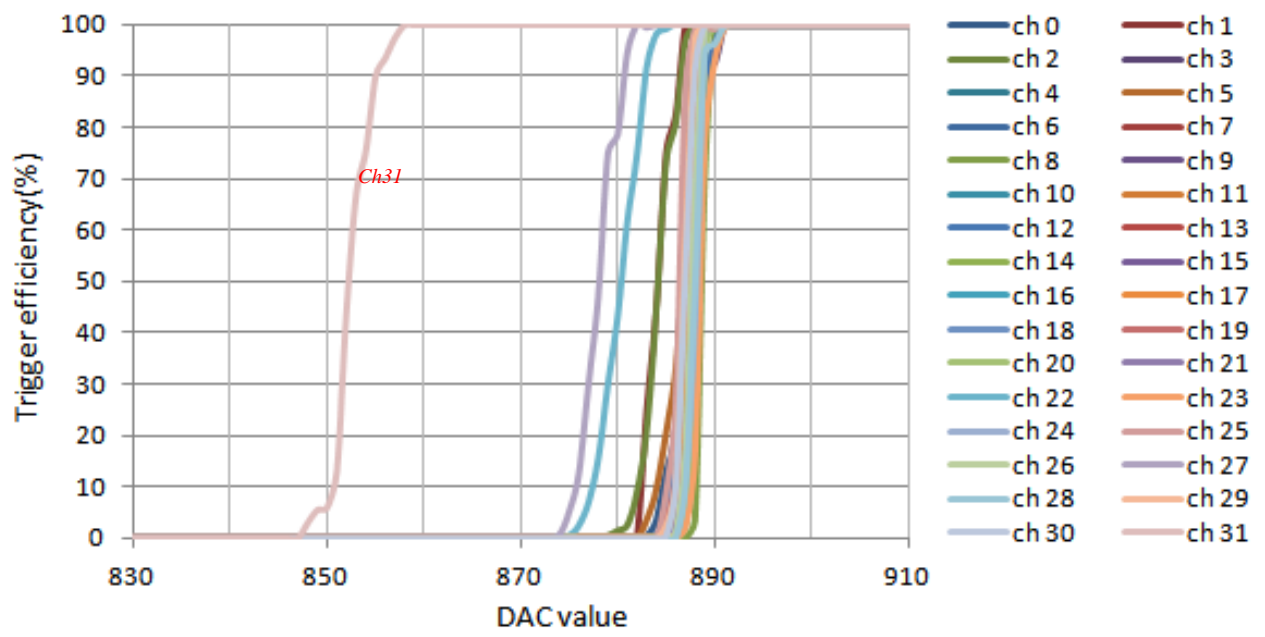

Figure 10: 32 channels s-curves

\section{Conclusion}

Achieving miniaturization of electronics cards with such dimensions is a real challenge. We tested all the cards and the measurements showed that all features needed for SIPMED are functioning well and that the performances (e.g DACs linearity, channels uniformity and $100 \%$ trigger efficiency) are suitable, except that the analog output signals are noisy, after investigation we noticed that the noise is caused by the third board. We are currently working to reduce noise for even better results.

\section{References}

[1] sales.hamamatsu.com/info/eul/MPPC/PDF/S11828-3344M_eng.pdf

[2] S. Callier : EASIROC, an easy \& versatile ReadOut device for SiPM, in Physics Procedia, 2OCT-2012, PHPRO2548. 